\title{
The Global Attractor of Thermoelastic Coupled System ${ }^{*}$
}

\author{
Danxia Wang, Jianwen Zhang ${ }^{\#}$ \\ Department of Mathematics, Taiyuan University of Technology, Taiyuan, China \\ Email: danxia.wang@163.com, \#jianwen.z2008@163.com
}

Received July 12, 2012; revised August 12, 2012; accepted September 12, 2012

\begin{abstract}
In this paper, we consider a class of Sine-Gordon equations which arise from the model of the thermoelastic coupled rod. Firstly, by virtue of the classical semigroup theory, we prove the existence and uniqueness of the mild solution under certain initial-boundary value for above-mentioned equations. Secondly, we obtain the boundedness of solutions by the priori estimates. Lastly, we prove the existence of a global attractor.
\end{abstract}

Keywords: The Mild Solution; Priori Estimates; Thermoelastic Coupled Rod; Global Attractor

\section{Introduction}

In this paper, we consider the following nonlinear thermoelastic coupled rod system

$$
\left\{\begin{array}{l}
u_{t t}-\Delta u-\alpha \Delta u_{t}+\gamma \sin u+\theta+u_{t}+\varepsilon u=g_{1}(x), \\
x \in \Omega, t \geq 0 \\
\theta_{t}-k \Delta \theta+u_{t}=g_{2}(x), x \in \Omega, t \geq 0 \\
\left.u(x, t)\right|_{x \in \partial \Omega}=\left.\theta(x, t)\right|_{x \in \partial \Omega}=0 \\
u(x, 0)=u_{0}(x), u_{t}(x, 0)=u_{1}(x), \theta(x, 0)=\theta_{0}(x), x \in \Omega
\end{array}\right.
$$

The above system describes the vibrations of an extensible thermoelastic rod model. Here $u=u(x, t)$ and $\theta=\theta(x, t)$ are all real-valued functions on $\Omega \times[0,+\infty]$, $\Omega=(0,1)$ is an open bounded domain of $R$. The coefficient $\alpha, \gamma, \varepsilon, k$ are all positive constants, where $\alpha$ is called the strong damping coefficient of rod, $\varepsilon$ is the small parameter. The sign $\Delta$ denotes

$$
\frac{\partial^{2}}{\partial x^{2}}, g_{1}(x), g_{2}(x) \in H_{0}^{1}(\Omega) \text {. }
$$

"Global solutions" and "global attractor" are two basic concepts in the study of long-time behavior of nonlinear dissipative evolution equations with various dissipation. If the coupled terms are equivalent to 0, Equations (1) will decouple to the Sine-Gordon equation

$$
u_{t t}-\Delta u-\alpha \Delta u_{t}+\gamma \sin u+u_{t}+\varepsilon u=g_{1}(x)
$$

and the heat equation. The structure of global attractors

\footnotetext{
${ }^{*}$ This work is supported by Natural Science Foundation of Shanxi Provice, and the Natural Science Foundation for Young Scientists of Shanxi Province (2010011008, 2011021002-2).

${ }^{\#}$ Corresponding author.
}

for weakly damped nonlinear wave Equation (2) as $\alpha, \varepsilon=$ 0 is studied in Temam [1] and Zhu [2] and Wang [3] and the one for the strongly damped nonlinear wave equation is considered in Zhou [4]. Semion [5] shows the Frechet differentiability for a damped sine-Gordon equation with a variable diffusion coefficient. Han [6] proves the existence of Random attractors for stochastic Sine-Gordon lattice system. But have the global solution and the global attractor for the "thermoelastic coupled" rod system (1)? To our knowledge, nothing was known until now.

In this paper, we give the proof of the existence and uniqueness of the mild solution and the existence of a global attractor for system (1) in space $H_{0}^{1}(\Omega) \times L^{2}(\Omega) \times L^{2}(\Omega)$.

\section{Existence and Uniqueness of Global Solutions}

It is well known that operator $A=-\Delta: D(A)=H_{0}^{1}(\Omega) \cap H^{2}(\Omega) \rightarrow L^{2}(\Omega)$ is self-adjoint, positive and linear and its eigenvalues $\left\{\lambda_{i}\right\}_{i \in N}$ satisfy $0<\lambda_{1} \leq \lambda_{2} \leq \cdots \leq \lambda_{m} \leq \cdots$ and $\lambda_{m} \rightarrow+\infty$ as $m \rightarrow+\infty$. Set $L^{2}(\Omega), H_{0}^{1}(\Omega), H^{2}(\Omega) \cap H_{0}^{1}(\Omega)$, $E=H_{0}^{1}(\Omega) \times L^{2}(\Omega) \times L^{2}(\Omega)$ with the usual inner products and norms, respectively

$$
\begin{gathered}
(u, v)=\int_{\Omega} u v \mathrm{~d} x,\|u\|=(u, u)^{\frac{1}{2}}, \forall u, v \in L^{2}(\Omega), \\
(u, v)_{1}=\int_{\Omega} \nabla u \nabla v \mathrm{~d} x,\|u\|_{1}=(u, u)_{1}^{\frac{1}{2}}, \forall u, v \in H_{0}^{1}(\Omega) \\
\left(y_{1}, y_{2}\right)_{E}=\left(u_{1}, u_{2}\right)_{1}+\left(v_{1}, v_{2}\right)+\left(\theta_{1}, \theta_{2}\right),\|y\|_{E}=(y, y)_{E}^{\frac{1}{2}} \\
\forall y_{i}=\left(u_{i}, v_{i}, \theta_{i}\right)^{T}, y=(u, v, \theta)^{T} \in E, i=1,2
\end{gathered}
$$

It is convenient to reduce (1) to an evolution equation 
of the first order in time, Let $u_{t}=v$, then (1) are equivalent to the following initial value problem in $E$,

$$
\left\{\begin{array}{l}
\frac{\mathrm{d} Y}{\mathrm{~d} t}=C Y+F(Y, t), \forall t \geq 0 \\
Y(0)=Y_{0}=\left(u_{0}, u_{1}, \theta_{0}\right)^{T} \in E
\end{array}\right.
$$

where $Y=(u, v, \theta)^{T}$,

$$
\begin{gathered}
F(Y, t)=\left(\begin{array}{c}
0 \\
-\gamma \sin u-u_{t}-\varepsilon u-\theta+g_{1}(x) \\
-u_{t}+g_{2}(x)
\end{array}\right) \\
C=\left(\begin{array}{ccc}
0 & I & 0 \\
\Delta & \alpha \Delta & 0 \\
0 & 0 & k \Delta
\end{array}\right)=\left(\begin{array}{ccc}
0 & I & 0 \\
-A & -\alpha A & 0 \\
0 & 0 & -k A
\end{array}\right)
\end{gathered}
$$

with $D(C)=D(A) \times D(A) \times D(A), I$ is the identity operator $L^{2}(\Omega), H_{0}^{1}(\Omega)$ and $D(A)$. Set $B=-C$, then similar to [7], by making some slight modification and reasoning, we can prove that for any $\alpha, k>0, B$ is a sectorial operator on $E$ and generates an analytic semigroup $e^{C t}$ on $E$ for $t>0$. By the assumptions $g_{1}(x), g_{2}(x) \in H_{0}^{1}(\Omega)$, it is easy to check that the function $F(Y, t): E \rightarrow E$ is locally Lipschitz continuous with respect to $Y$, By the classical semigroup theory concerning the existence and uniqueness of the solution of evolution differential equations, we have

Theorem 2.1. Assume that the assumptions $g_{1}(x)$, $g_{2}(x) \in H_{0}^{1}(\Omega)$ hold, then consider the initial value problem (3) in Hilbert space $E$. For any initial value $Y_{0}=\left(u_{0}, u_{1}, \theta_{0}\right)^{T} \in E$, there exists a unique continuous function $\left.Y(t)=Y\left(t, Y_{0}\right)=(u, v, \theta)^{T} \in C((0,+\infty)) ; E\right)$ such that $Y(t)=(u(t), v(t), \theta(t))^{T}$ satisfies the integral equation

$$
Y(t)=Y\left(t, Y_{0}\right)=e^{C t} Y_{0}+\int_{0}^{t} e^{C(t-s)} F(Y(s), s) \mathrm{d} s .
$$

In this case, $Y(t)$ is called a mild solution of the system (3) and $Y(t)=Y\left(t, Y_{0}\right)$ is jointly continuous in $t$ and $Y_{0}$, that is, the solution $\left(u, u_{t}, \theta\right)$ of the system (1) satisfies $\left.\left(u, u_{t}, \theta\right) \in C((0,+\infty)), E\right)$.

By Theorem 2.1, for any $t>0$, we may introduce the map

$$
\{S(t), t \geq 0\}:\left\{u_{0}, u_{1}, \theta_{0}\right\} \rightarrow\left\{u(t), u_{t}(t), \theta(t)\right\}, E \rightarrow E .
$$

It maps $E=H_{0}^{1}(\Omega) \times L^{2}(\Omega) \times L^{2}(\Omega)$ into itself and it enjoys the usual process properties as follows

$$
\left\{\begin{array}{l}
S(0)=I \\
S(t+\tau)=S(t) S(\tau), \forall t \geq 0
\end{array}\right.
$$

It is obviously that the map $\{S(t), t>0\}, \forall t \in R$ is continuous in $E$. In the following, we will introduce the existence of the bounded absorbing set and the global attractor for map $\{S(t), t>0\}$ in $E$.

\section{The Existence of Bounded Absorbing Set}

In this section, we will show boundedness of the solutions for system (3). For this purpose, we define a weighted inner product and norm in

$E=H_{0}^{1}(\Omega) \times L^{2}(\Omega) \times L^{2}(\Omega)$ by

$$
\begin{aligned}
& \left(\varphi_{1}, \varphi_{2}\right)=\mu\left(u_{1}, u_{2}\right)_{1}+\left(w_{1}, w_{2}\right)+\left(\theta_{1}, \theta_{2}\right), \\
& \|\varphi\|_{E}=(\varphi, \varphi)_{E}^{\frac{1}{2}}
\end{aligned}
$$

for any $\varphi_{i}=\left(u_{i}, w_{i}, \theta_{i}\right)^{T} \in E, i=1,2$, where $w_{i}=u_{i t}+\varepsilon_{i}$, $i=1,2$ and $\mu$ is chosen by

$$
\mu=\frac{2+\alpha^{2}}{2+2 \alpha^{2}}
$$

Obviously the norm $\|\cdot\|_{E}$ in (4) is equivalent to the usual norm in space $E$.

Let $\varphi=(u, w, \theta)^{T}, w=u_{t}+\varepsilon u$, where $\varepsilon$ is chosen as

$$
\varepsilon=\frac{\alpha}{2+2 \alpha^{2}}
$$

then the system (3) can be written as

$$
\begin{aligned}
& \varphi_{t}+H(\varphi)=F_{1}(\varphi, t), \\
& \varphi(0)=\left(u_{0}, w_{0}=u_{1}+\varepsilon u_{0}, \theta_{0}\right), t \geq 0
\end{aligned}
$$

$$
\begin{aligned}
& \text { where } F_{1}(\varphi, t)=\left(\begin{array}{c}
0 \\
-\gamma \sin u+g_{1}(x) \\
g_{2}(x)
\end{array}\right) \\
& \text { and } H(\varphi)=\left(\begin{array}{c}
\varepsilon u-w \\
A u+\theta-\varepsilon(w-\varepsilon u)+\alpha A(w-\varepsilon u)+w \\
k A \theta+w-\varepsilon u
\end{array}\right)
\end{aligned}
$$

Obviously, the mapping

$$
\begin{aligned}
& S_{\varepsilon}(t):\left(u_{0}, u_{1}+\varepsilon u_{0}, \theta_{0}\right)^{T} \rightarrow\left(u, u_{t}+\varepsilon u, \theta\right)^{T}, \\
& E \rightarrow E, t \geq 0
\end{aligned}
$$

has the relation with

$$
S(t): S_{\varepsilon}(t)=R_{\varepsilon} S(t) R_{-\varepsilon}
$$

where $R_{\varepsilon}\{u, w, \theta\} \rightarrow\{u, w+\varepsilon u, \theta\}$ is an isomorphism of $E$. So we only need consider the equivalent map (6).

For the boundedness of the solution of (5) in $E$, we firstly present an important lemma which plays an important role in this artical.

Lemma 3.1. For any $\varphi=(u, w, \theta)^{T} \in H_{0}^{1}(\Omega) \times L^{2}(\Omega) \times L^{2}(\Omega)$. we have

$$
(H(\varphi), \varphi)_{E} \geq \delta_{1}\|\varphi\|_{E}^{2}+\delta_{2}\|w\|^{2}+\delta_{3}\|\theta\|^{2}+\alpha\left\|A^{\frac{1}{2}} w\right\|^{2}
$$

where 


$$
\delta_{1}=\varepsilon-\frac{\varepsilon^{3}}{4 \mu}, \delta_{2}=1-4 \varepsilon+\frac{\varepsilon^{3}}{4 \mu}, \delta_{3}=2 k-\varepsilon+\frac{\varepsilon^{3}}{4 \mu}-\frac{2}{\varepsilon}
$$

are all positive constants if $k>\frac{\varepsilon}{2}+\frac{1}{\varepsilon}$.

\section{Proof.}

$$
\begin{aligned}
& (H(\varphi), \varphi)_{E}-\left(\delta_{1}\|\varphi\|_{E}^{2}+\delta_{2}\|w\|^{2}+\delta_{3}\|\theta\|^{2}+\alpha\left\|A^{\frac{1}{2}} w\right\|^{2}\right) \\
= & \mu\left(\varepsilon-\delta_{1}\right)\|u\|_{1}^{2}+\left(1-\varepsilon-\delta_{1}-\delta_{2}\right)\|w\|^{2} \\
& +\left(2 k-\delta_{1}-\delta_{3}\right)\|\theta\|^{2}-2(w, \theta)-\varepsilon(u, \theta)+\varepsilon^{2}(u, w) \\
\geq & \mu\left(\varepsilon-\delta_{1}\right)\|u\|_{1}^{2}+\left(1-\varepsilon-\delta_{1}-\delta_{2}\right)\|w\|^{2}+\left(2 k-\delta_{1}-\delta_{3}\right) \\
& \times\|\theta\|^{2}-2\|\theta\|\|w\|-\varepsilon\|u\|\|\theta\|-\varepsilon^{2}\|u\|\|w\| \\
\geq & \mu\left(\varepsilon-\delta_{1}\right)\|u\|^{2}-\varepsilon^{2}\|u\|\|w\|+\frac{1-\varepsilon-\delta_{1}-\delta_{2}}{2}\|w\| \\
& +\mu\left(\varepsilon-\delta_{1}\right)\|u\|^{2}-\varepsilon\|u\|\|\theta\|+\frac{2 k-\delta_{1}-\delta_{3}}{2}\|\theta\|^{2} \\
& +\frac{1-\varepsilon-\delta_{1}-\delta_{2}}{2}\|w\|^{2}-2\|\theta\|\|w\|+\frac{2 k-\delta_{1}-\delta_{3}}{2}\|\theta\|^{2}
\end{aligned}
$$

with

$$
\begin{gathered}
2 \mu\left(\varepsilon-\delta_{1}\right)\left(1-\varepsilon-\delta_{1}-\delta_{2}\right)=\varepsilon^{4} \\
2 \mu\left(\varepsilon-\delta_{1}\right)\left(2 k-\delta_{1}-\delta_{3}\right)=\varepsilon^{2} \\
\left(1-\varepsilon-\delta_{1}-\delta_{2}\right)\left(2 k-\delta_{1}-\delta_{3}\right)=4
\end{gathered}
$$

the Lemma is easily obtained.

Theorem 3.1. There exists a positive constant $M>0$ such that for any bounded set $B$ of $E$, then there exists $T_{0}(B) \geq 0$ such that the solution $\varphi=(u(t), w(t), \theta(t))^{T}$ of (5) with $\varphi(0) \in B$ satisfies

$$
\begin{aligned}
& \|\varphi(t)\|_{E}=\left(\mu\|u(t)\|_{1}^{2}+\|w(t)\|^{2}+\|\theta(t)\|^{2}\right)^{\frac{1}{2}} \leq M, \\
& \forall t \geq T_{0}(B)
\end{aligned}
$$

in which $w=u_{t}+\varepsilon u$.

Proof. Let $\varphi=(u, w, \theta)^{T}, w=u_{t}+\varepsilon u$ be the solution of (5) with initial value $\phi(0)=\left(\left(u_{0}, w_{0}, \theta_{0}\right)\right)^{T} \in E$. Taking the inner product $(\cdot, \cdot)_{E}$ of (5) with $\varphi=(u, w, \theta)^{T}$ and considering of Lemma 3.1, we have

$$
\begin{aligned}
& \frac{1}{2} \frac{d}{d t}\|\varphi(t)\|_{E}^{2}+\delta_{1}\|\varphi(t)\|_{E}^{2}+\delta_{2}\|w\|^{2} \\
& +\delta_{3}\|\theta\|^{2}+\alpha\left\|A^{\frac{1}{2}} w\right\|^{2} \leq\left(F_{1}(\varphi, t), \varphi\right)_{E}
\end{aligned}
$$

with

$$
\left(F_{1}(\varphi, t), \varphi\right)_{E} \leq \frac{\gamma^{2}}{8 \alpha}+2 \alpha\|w\|^{2}+\frac{1}{4 \delta_{2}}\left\|g_{1}(x)\right\|^{2}
$$

$$
+\delta_{2}\|w\|^{2}+\frac{1}{4 \delta_{3}}\left\|g_{2}(x)\right\|^{2}+\delta_{3}\|\theta\|^{2},
$$

we have

$$
\begin{aligned}
& \frac{1}{2} \frac{\mathrm{d}}{\mathrm{d} t}\|\varphi(t)\|_{E}^{2}+\delta_{1}\|\varphi(t)\|_{E}^{2} \\
\leq & \frac{\gamma^{2}}{8 \alpha}+\left.\frac{1}{4 \delta_{2}}\left\|g_{1}(x)\right\|\right|^{2}+\frac{1}{4 \delta_{3}}\left\|g_{2}(x)\right\|^{2}
\end{aligned}
$$

By Gronwall's inequality we have

$$
\begin{aligned}
& \|\varphi(t)\|_{E}^{2} \leq\left\|\varphi_{0}\right\|_{E}^{2} e^{-2 \delta_{1} t}+\frac{1}{2 \delta_{1}}\left(\frac{\gamma^{2}}{4 \alpha}+\frac{1}{2 \delta_{2}}\left\|g_{1}\right\|^{2}+\frac{1}{2 \delta_{3}}\left\|g_{2}\right\|^{2}\right) \\
& \text { Set } \quad M^{2}=\frac{1}{\delta_{1}}\left(\frac{\gamma^{2}}{4 \alpha}+\frac{1}{2 \delta_{2}}\left\|g_{1}\right\|^{2}+\frac{1}{2 \delta_{3}}\left\|g_{2}\right\|^{2}\right)
\end{aligned}
$$

thus we complete the proof of the Theorem.

Corollary 3.1. Let $B_{0}$ be a bounded closed ball of $E$ centered at 0 of radius $M$. For any initial value $\|\varphi(0)\|_{E}^{2} \leq M^{2}$, there exists a constant $M_{1}=M_{1}(M)$ such that the solution of (5) $\varphi(t)=(u(t), w(t), \theta(t))^{T}$ satisfies $\|\varphi(t)\|_{E}^{2} \leq M_{1}(M), \forall t \geq 0$.

\section{The Existence of a Global Attractor}

Theorem 4.1. For any initial value $\varphi_{0} \in B_{0}$, the solution $\varphi(t)=(u(t), w(t), \theta(t))^{T}$ of (5) can be decomposed into $\varphi(t)=\varphi_{1}(t)+\varphi_{2}(t)$ where $\varphi_{i}(t)=\left(u_{i}(t), w_{i}(t), \theta_{i}(t)\right)^{T}$, $w_{i}(t)=u_{i t}(t)+\varepsilon u_{i}(t), i=1,2$ satisfy, respectively $(\forall t>0)$

$$
\begin{gathered}
\left\|\varphi_{1}(t)\right\|_{E}^{2}=\mu\left\|u_{1}(t)\right\|_{1}^{2}+\left\|w_{1}(t)\right\|^{2}+\left\|\theta_{1}(t)\right\|^{2} \leq M_{2}^{2} e^{-2 \delta_{1} t} \\
\left\|A^{\frac{1}{2}} \varphi_{2}(t)\right\|_{E}^{2}=\mu\left\|A^{\frac{1}{2}} u_{2}(t)\right\|_{1}^{2}+\left\|A^{\frac{1}{2}} w_{2}(t)\right\|^{2}+\left\|A^{\frac{1}{2}} \theta_{2}(t)\right\|^{2} \\
\leq M_{3}^{2}
\end{gathered}
$$

where $M_{2}^{2}$ and $M_{3}^{2}$ depend on $M$.

Proof. Let $\varphi(t)=(u(t), w(t), \theta(t))^{T}, \forall t \geq 0$ be a solution of (5) in space $E$ with the initial value $\varphi(0)=\left(u_{0}, w_{0}, \theta_{0}\right)^{T} \in B_{0}$.

Let $\varphi(t)=\varphi_{1}(t)+\varphi_{2}(t)$, where $\varphi_{i}(t)=\left(u_{i}(t), w_{i}(t), \theta_{i}(t)\right)^{T}$, $w_{i}(t)=u_{i t}(t)+\varepsilon u_{i}(t), i=1,2$, satisfy, respectively

$$
\left\{\begin{array}{l}
\varphi_{1 t}+H\left(\varphi_{1}\right)=0, \forall t \geq 0 \\
\varphi_{1}(0)=\left(u_{1}(0), w_{1}(0), \theta_{1}(0)\right)^{T}=\left(u_{0}, w_{0}, \theta_{0}\right)^{T} \in E
\end{array}\right.
$$

where

$$
H\left(\varphi_{1}\right)=\left(\begin{array}{c}
\varepsilon u_{1}-w_{1} \\
A u_{1}+\theta_{1}-\varepsilon\left(w_{1}-\varepsilon u_{1}\right)+\alpha A\left(w_{1}-\varepsilon u_{1}\right)+w_{1} \\
k A \theta_{1}+w_{1}-\varepsilon u_{1}
\end{array}\right)
$$

and

$$
\left\{\begin{array}{l}
\varphi_{2 t}+H\left(\varphi_{2}\right)=F_{1}(\varphi, t), \forall t \geq 0 \\
\varphi_{2}(0)=\left(u_{2}(0), w_{2}(0), \theta_{2}(0)\right)^{T}=(0,0,0)^{T} \in E
\end{array}\right.
$$


where

$$
H\left(\varphi_{2}\right)=\left(\begin{array}{c}
\varepsilon u_{2}-w_{2} \\
A u_{2}+\theta_{2}-\varepsilon\left(w_{2}-\varepsilon u_{2}\right)+\alpha A\left(w_{2}-\varepsilon u_{2}\right)+w_{2} \\
k A \theta_{2}+w_{2}-\varepsilon u_{2}
\end{array}\right)
$$

Taking the inner product of (9) with $\varphi_{1}(t)$ we have

$$
\frac{1}{2} \frac{\mathrm{d}}{\mathrm{d} t}\left\|\varphi_{1}\right\|_{E}^{2}+\left(H\left(\varphi_{1}\right), \varphi_{1}\right)_{E}=0
$$

Similar to the proof of Lemma 3.1, we can obtain

$$
\left(H\left(\varphi_{1}\right), \varphi_{1}\right)_{E} \geq \delta_{4}\left\|\varphi_{1}\right\|_{E}^{2}
$$

where $\quad \delta_{4}=\delta_{1}=\varepsilon-\frac{\varepsilon^{3}}{4 \mu}>0$.

Thus by (12) and Gronwall's inequality, (7) is easily obtained from (11).

By putting $x=0,1$ and $t=0$ in (10) we get

$$
u_{2 x x t}(0,0)=u_{2 x x t}(1,0)=0
$$

The operator $A^{\frac{1}{2}}$ operates into (10) to get

$$
\left\{\begin{array}{l}
A^{\frac{1}{2}} \varphi_{2 t}+A^{\frac{1}{2}} H\left(\varphi_{2}\right)=A^{\frac{1}{2}} F_{1}(\varphi, t), \\
A^{\frac{1}{2}} \varphi_{2}(0)=A^{\frac{1}{2}}\left(u_{2}(0), w_{2}(0), \theta_{2}(0)\right)^{T}=(0,0,0)^{T} \in E
\end{array}\right.
$$

Taking the inner product of (14) with

$$
\begin{aligned}
& A^{\frac{1}{2}} \varphi_{2}=\left(A^{\frac{1}{2}} u_{2}, A^{\frac{1}{2}} w_{2}, A^{\frac{1}{2}} \theta_{2}\right)^{T} \\
& \frac{1}{2} \frac{\mathrm{d}}{\mathrm{d} t}\left\|A^{\frac{1}{2}} \varphi_{2}\right\|_{E}^{2}+\left(A^{\frac{1}{2}} H\left(\varphi_{2}\right), A^{\frac{1}{2}} \varphi_{2}\right)_{E}=\left(A^{\frac{1}{2}} F_{1}(\varphi, t), A^{\frac{1}{2}} \varphi_{2}\right)_{E}
\end{aligned}
$$

Considering to (13) we get that

$$
\begin{aligned}
& \left(A^{\frac{1}{2}} H\left(\varphi_{2}\right), A^{\frac{1}{2}} \varphi_{2}\right)_{E} \\
= & \mu \varepsilon\left\|A^{\frac{1}{2}} u_{2}\right\|_{1}^{2}+\alpha\left\|A w_{2}\right\|^{2}+\left\|A^{\frac{1}{2}} w_{2}\right\|^{2} \varphi \\
+ & k\left\|A \theta_{2}\right\|^{2}+2\left(A^{\frac{1}{2}} w_{2}, A^{\frac{1}{2}} \theta_{2}\right)-\varepsilon\left(A^{\frac{1}{2}} u_{2}, A^{\frac{1}{2}} \theta_{2}\right) \\
- & \varepsilon\left\|A^{\frac{1}{2}} w_{2}\right\|^{2}+\varepsilon^{2}\left(A^{\frac{1}{2}} u_{2}, A^{\frac{1}{2}} w_{2}\right)
\end{aligned}
$$

with

$$
\begin{aligned}
& \left(A^{\frac{1}{2}} F_{1}(\varphi, t), A^{\frac{1}{2}} \varphi_{2}\right)_{E} \\
\leq & \frac{\gamma^{2}}{4 \alpha}+\alpha\left\|A w_{2}\right\|^{2}+\left(A^{\frac{1}{2}} g_{1}(x, t), A^{\frac{1}{2}} w_{2}\right) \\
& +\left(A^{\frac{1}{2}} g_{2}(x, t), A^{\frac{1}{2}} \theta_{2}\right)
\end{aligned}
$$

from (15) we have

$$
\begin{aligned}
& \frac{1}{2} \frac{\mathrm{d}}{\mathrm{d} t}\left\|A^{\frac{1}{2}} \varphi_{2}\right\|_{E}^{2}+\mu \varepsilon\left\|A^{\frac{1}{2}} u_{2}\right\|_{1}^{2}+(1-\varepsilon)\left\|A^{\frac{1}{2}} w_{2}\right\|^{2}+2 k\left\|A^{\frac{1}{2}} \theta_{2}\right\|^{2} \\
& +2\left(A^{\frac{1}{2}} w_{2}, A^{\frac{1}{2}} \theta_{2}\right)-\varepsilon\left(A^{\frac{1}{2}} u_{2}, A^{\frac{1}{2}} \theta_{2}\right)+\varepsilon^{2}\left(A^{\frac{1}{2}} u_{2}, A^{\frac{1}{2}} w_{2}\right) \\
& \leq \frac{\gamma^{2}}{4 \alpha}+\left(A^{\frac{1}{2}} g_{1}(x, t), A^{\frac{1}{2}} w_{2}\right)+\left(A^{\frac{1}{2}} g_{2}(x, t), A^{\frac{1}{2}} \theta_{2}\right)
\end{aligned}
$$

Similar to the proof of the Lemma 3.1, from (16) we get

$$
\begin{aligned}
& \frac{1}{2} \frac{\mathrm{d}}{\mathrm{d} t}\left\|A^{\frac{1}{2}} \varphi_{2}\right\|_{E}^{2}+2 \delta_{1}\left\|A^{\frac{1}{2}} \varphi_{2}\right\|_{E}^{2} \\
\leq & \frac{\gamma^{2}}{2 \alpha}+\frac{1}{2 \delta_{2}}\left\|A^{\frac{1}{2}} g_{1}\right\|^{2}+\frac{1}{2 \delta_{3}}\left\|A^{\frac{1}{2}} g_{2}\right\|^{2}
\end{aligned}
$$

By the Gronwall's inequality, we have

$$
\begin{aligned}
& \left\|A^{\frac{1}{2}} \varphi_{2}(t)\right\|_{E}^{2} \leq\left\|A^{\frac{1}{2}} \varphi_{2}(0)\right\|_{E}^{2} e^{\left(-2 \delta_{1} t\right)} \\
& +\frac{1}{2 \delta_{1}}\left(\frac{\gamma^{2}}{2 \alpha}+\frac{1}{2 \delta_{2}}\left\|A^{\frac{1}{2}} g_{1}\right\|^{2}+\frac{1}{2 \delta_{3}}\left\|A^{\frac{1}{2}} g_{2}\right\|^{2}\right)
\end{aligned}
$$

Set

$$
M_{3}^{2}=\frac{1}{\delta_{1}}\left(\frac{\gamma^{2}}{2 \alpha}+\frac{1}{2 \delta_{2}}\left\|A^{\frac{1}{2}} g_{1}\right\|^{2}+\frac{1}{2 \delta_{3}}\left\|A^{\frac{1}{2}} g_{2}\right\|^{2}\right) .
$$

Thus, the proof of Theorem 4.1 is complete.

\section{REFERENCES}

[1] R. Temam, "Infinite-Dimensional Dynamical Systems in Mechanics and Physics," Applied Mathematical Sciences, Vol. 68, Springer-Verlag, New York, 1988.

[2] S. Zhu and S. F. Zhou, "Dimension of the Global Attractor for the Damped and Driven SINE-Gordon Equation," Nonlinear Analysis, Vol. 37, 1999, pp. 389-399.

[3] G. X. Wang and S. Zhu, "Dimension of the Global Attractor for the Discretized Damped Sine-Gordon Equation," Applied Mathematics and Computation, Vol. 117, No. 2-3, 2001, pp. 257-265. doi:10.1016/S0096-3003(99)00179-4

[4] S. F. Zhou, "Dimension of the Global Attractor for Strongly Damped Nonlinear Wave Equation," Journal of Mathematical Analysis and Applications, Vol. 233, No. 1, 1999, pp. 102-115. doi:10.1006/jmaa.1999.6269

[5] G. Semion, "Frechet Differentiability for a Damped SineGordon Equation," Journal of Mathematical Analysis and Applications, Vol. 360, No. 2, 2009, pp. 503-517. doi:10.1016/j.jmaa.2009.06.074

[6] X. Y. Han, "Randon Attractors for Stochastic Sine-Gordon Lattice Systems with Multiplicative White Noise," Journal of Mathematical Analysis and Applications, Vol. 376, No. 2, 2011, pp. 481-493. doi:10.1016/j.jmaa.2010.11.032 
[7] P. Massatt, "Limiting Behavior for Strongly Damped Nonlinear Wave Equations," Journal of Differential Equa- tions, Vol. 48, No. 3, 1983, pp. 334-349. doi:10.1016/0022-0396(83)90098-0 